\title{
FACTORS THAT INFLUENCE THE DEVELOPMENT OF ATRIAL FLUTTER AFTER THE FONTAN OPERATION
}

Steven B. Fishberger, MD ${ }^{\mathrm{a} *}$

Gil Wernovsky, MD ${ }^{\mathrm{a} * *}$

Thomas L. Gentles, MB, $\mathrm{ChB}^{\mathrm{a} * * *}$

Kimberlee Gauvreau, ScD ${ }^{\mathrm{a}}$

Janice Burnett ${ }^{\mathbf{a}}$

John E. Mayer, Jr., MD ${ }^{b}$

Edward P. Walsh, MD ${ }^{\mathrm{a}}$
Objectives: Atrial flutter is a frequent, potentially fatal complication of the Fontan operation, but risk factors for its development are ill defined. We evaluated clinical features that might predict the development of atrial flutter in patients who had a Fontan operation. Methods: We evaluated 334 early survivors of a Fontan operation done between April 1973 and July 1991 (mean follow-up, $5.0 \pm 3.8$ years). Evaluation included electrocardiography, Holter monitor recordings, and chart review. Modifications of the Fontan operation included an extracardiac conduit $(n=43)$, an atriopulmonary anastomosis $(n=117)$, or a total cavopulmonary anastomosis $(n=$ 174). Patient, time, and procedure-related variables were analyzed with respect to the development of atrial flutter. Results: Atrial flutter was identified in $54(16 \%)$ patients at a mean of $5.3 \pm 4.7$ years (range 0 to 19.7 years) after Fontan operation. Atrial flutter developed sooner and was more likely to occur in patients who were older at the time of Fontan operation $(12.4 \pm 7.6$ vs $6.3 \pm 5.2$ years; $p<0.001)$, had a longer follow-up interval $(8.7 \pm 3.9$ vs $4.4 \pm 3.4$ years; $p<0.001)$, had a prior atrial septectomy or pulmonary artery reconstruction $(p<0.01$ ), and had worse New York Heart Association class symptoms $(p<0.02)$. The presence of sinus node dysfunction was associated with a higher incidence of atrial flutter $(p<$ 0.001). Although there was a lower prevalence of atrial flutter in those patients with a total cavopulmonary anastomosis, the follow-up for this group was shorter. Anatomic diagnoses, perioperative hemodynamics, and other previous palliative operations were not associated with an increased incidence of atrial flutter. Multivariate analysis identified age at operation, duration of follow-up, extensive atrial baffling, and type of repair as factors associated with the development of atrial flutter after Fontan operation. Conclusion: Atrial flutter continues to develop with time after the Fontan operation. Further follow-up is necessary to determine whether a total cavopulmonary anastomosis reduces the incidence of atrial flutter. (J Thorac Cardiovasc Surg 1997;113:80-6)

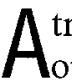
trial flutter is a frequent sequela of the Fontan operation with a reported prevalence of $12.5 \%$ to $26 \%$ at intermediate and late follow-up. ${ }^{1-5}$ Atrial flutter in a patient with a Fontan circulation can cause significant hemodynamic compromise and is

From the Departments of Cardiology and Cardiac Surgery, ${ }^{\mathrm{b}}$ Children's Hospital; and the Departments of Pediatrics and Surgery, Harvard Medical School, Boston, Mass.

Supported in part by the Kobren Fund.

Received for publication Jan. 15, 1996; revisions requested Feb. 19, 1996; revisions received May 30, 1996; accepted for publication July 18, 1996.

Address for reprints: Edward P. Walsh, MD, Department of Cardiology, Children's Hospital, 300 Longwood Ave., Boston, MA 02115. potentially fatal. ${ }^{1,3,6-8}$ The high prevalence of atrial arrhythmias may be a result of extensive atrial surgical procedures, elevated atrial pressures, and atrial enlargement. ${ }^{2-4,9}$ Atrial flutter is the single most common atrial arrhythmia in this patient pop-

\footnotetext{
*Current address: Division of Pediatric Cardiology, The Mount Sinai Medical Center, New York, N.Y. * Current address: Division of Pediatric Cardiology, Children's
Hospital of Philadelphia, Philadelphia, Pa.
***:Current address: Department of Cardiology, Greenlane Hos- pital, Aukland, New Zealand.

Copyright (C) 1997 by Mosby-Year Book, Inc. $0022-5223 / 97 \$ 5.00+0 \quad \mathbf{1 2 / 1 / 7 6 8 3 3}$ 
ulation $^{3-5}$; however, risk factors associated with this complication remain ill defined. Previous studies have been based on small numbers of patients ${ }^{2-4}$ or have combined a variety of supraventricular arrhythmias with different mechanisms. ${ }^{10}$

The purpose of this study was to evaluate clinical features that may predict the development of atrial flutter in a large group of patients who have undergone a Fontan operation and to identify factors predisposing to an earlier development of atrial flutter.

\section{Methods}

Patients. Between April 1973 and July 1991, 500 patients underwent a Fontan operation at Children's Hospital, Boston. A retrospective chart review and a review of all available electrocardiograms (ECGs)and Holter monitor recordings were done. As part of a comprehensive cross-sectional review, patients' cardiologists were requested to provide a recent clinical evaluation, ECG, and Holter monitor recording. Factors related to early and late outcome and hemodynamic findings are the subjects of separate reports.

Inclusion criteria for this study included early survival with a Fontan circulation and the availability of an ECG or Holter monitor recording at least 1 month after Fontan operation. Among the 500 patients, 84 had an early failure or death and were eliminated from the analysis. Of the 416 potentially eligible patients, 82 had inadequate follow-up information and were also excluded; therefore 334 patients form the basis of this report. An ECG was done in all patients, and 193 patients had a Holter monitor recording.

Definitions. For the purposes of this study, atrial flutter was defined as a documented primary intraatrial reentrant rhythm that occurred beyond the immediate postoperative period. The time to atrial flutter was defined as the time to the first episode documented by either an ECG or Holter monitor recording. Sinus node dysfunction was defined as being present if the patient had marked sinus bradycardia (resting heart rate $>2$ standard deviations less than age-adjusted mean), junctional rhythm, or symptomatic bradycardia resulting in placement of a pacemaker.

The types of Fontan procedure were grouped into three categories: an extracardiac conduit, a direct atriopulmonary connection, and a total cavopulmonary anastomosis. An extracardiac conduit used a Dacron tube connecting the right atrium to the right ventricular outflow tract or the pulmonary artery. The direct atriopulmonary connection was done by incising the roof of the right atrium and suturing it posteriorly to the right pulmonary artery. In some cases, this connection was augmented with a pericardial patch. The total cavopulmonary anastomosis was done with an end-to-side anastomosis of the cardiac and cephalic end of the superior vena cava to the right pulmonary artery. A polytetrafluoroethylene baffle was sutured along the lateral wall of the right atrium directing
Table I. Variables examined with respect to development of atrial flutter

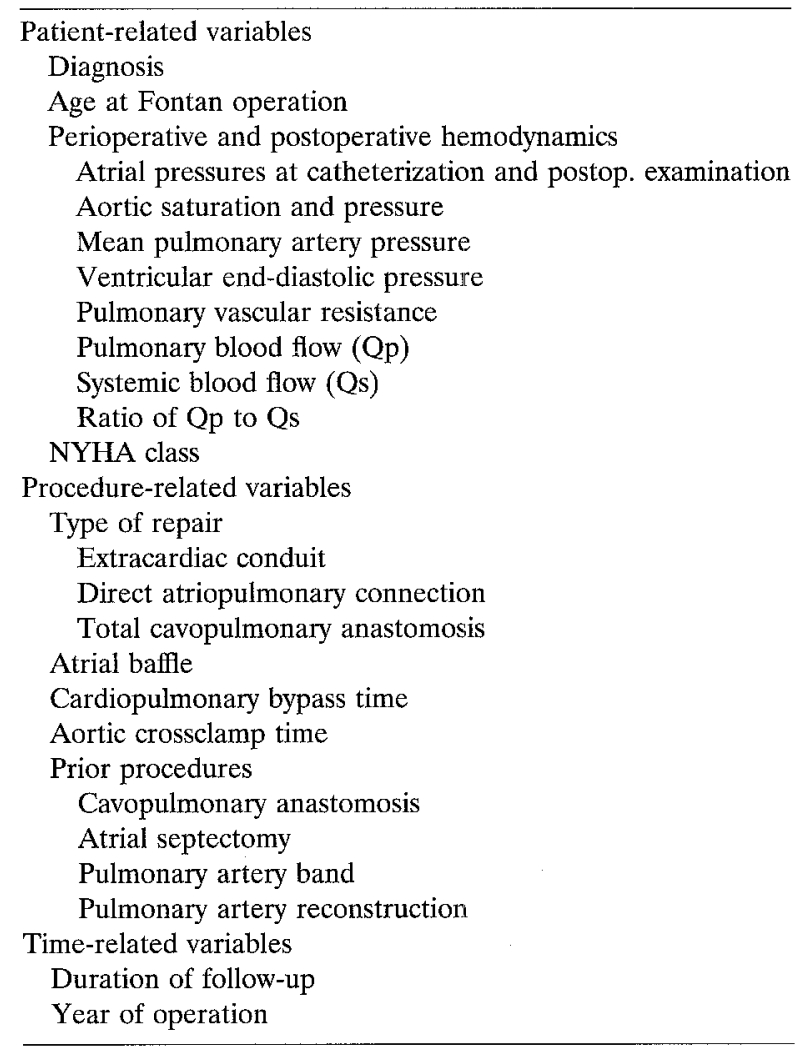

blood from the inferior vena cava to the superior vena cava.

Variables evaluated. Variables examined that were potentially associated with atrial flutter are shown in Table I and include patient-related, procedure-related, and timerelated variables. The presence of sinus node dysfunction in the postoperative period was noted.

Statistical analysis. The primary outcome variable in this study was time to the development of atrial flutter. Patients in whom atrial flutter did not develop were considered to be censored at the time of most-recent follow-up. Patients who did not have follow-up information pertaining to the onset of arrhythmia that was obtained at least 1 month after the Fontan operation were eliminated from the analysis. Potential selection bias was examined by comparing perioperative data from study patients with data from those who were excluded. Because patients who did not have more than 1 month of follow-up were excluded from the analysis, time to atrial flutter was defined as the period beginning 30 days after the Fontan procedure and extending until the onset of flutter. For patients in whom arrhythmia began less than 1 month after operation, failure time is taken to be zero. By defining time to atrial flutter in this way, the uncertain effects of early arrhythmia in patients who have no subsequent follow-up are eliminated. For categorical predictor variables, survival estimates were obtained for each 


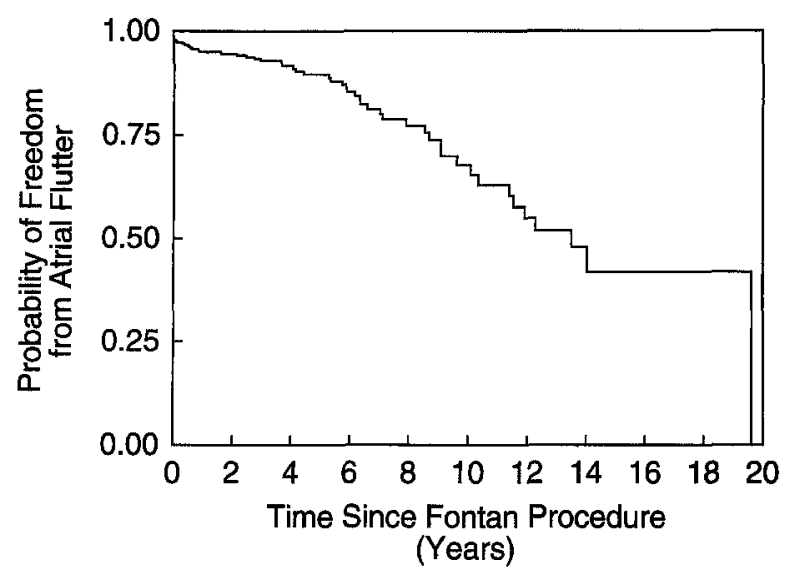

Fig. 1. Kaplan-Meier curve of freedom from atrial flutter as function of time since Fontan operation.

level of the variable with use of the Kaplan-Meier method; subgroups were then compared by the log-rank test. Continuous predictors were broken down into quartiles and evaluated in a similar fashion. To assess the simultaneous effects of these variables on time to atrial flutter, variables that were significant at the 0.10 level in a univariate analysis were included in a multivariate proportional-hazards model. A significance level of 0.05 was required for retention in the multivariate model.

In a secondary analysis, an indicator of atrial flutter was treated as a binary response variable. Univariate relationships with categorical predictors were examined by the $\chi^{2}$ test; relationships with continuous variables were evaluated by logistic regression. The simultaneous effects of these variables on the development of atrial flutter were assessed with use of a multivariate logistic regression model. These models controlled for duration of follow-up, because the longer a person is followed up the greater the opportunity for atrial flutter to develop and be observed.

\section{Results}

Study patients. The mean age at Fontan operation was $7.3 \pm 6.1$ years, with a mean follow-up of $5.0 \pm 3.8$ years. Anatomic diagnoses were grouped into six categories: 111 patients had a single left ventricle and normally related great arteries, 133 had a single left ventricle and transposed great arteries, 35 had a single right or unknown ventricle, 11 had hypoplastic left heart syndrome, 25 had heterotaxy syndrome, and 19 had other diagnoses. An extracardiac conduit was used in 43 patients, a direct atriopulmonary connection in 117 , and a total cavopulmonary anastomosis in 174 . During the follow-up period 309 patients have survived with a Fontan circulation, whereas 18 have died and 7 had either a heart transplantation $(n=5)$ or a takedown

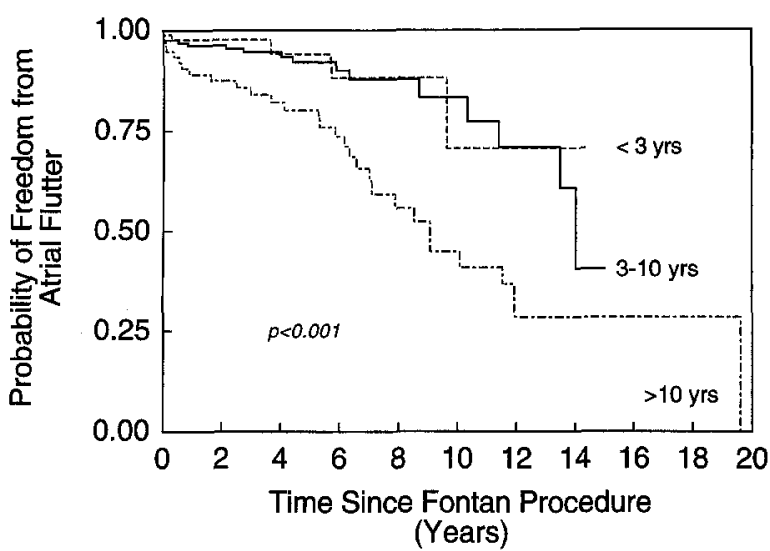

Fig. 2. Kaplan-Meier curves of freedom from atrial flutter as function of age at Fontan operation.

to a bidirectional Glenn $(n=1)$ or systemic-topulmonary artery shunt $(n=1)$.

When compared with the 334 patients in the study sample, the 82 late survivors who had inadequate follow-up and were therefore excluded did not differ with respect to age at operation, sex, anatomic diagnosis, type of repair, or preoperative hemodynamics.

Atrial flutter was identified in $54(16 \%)$ patients. The first episode of atrial flutter was identified at a mean of $5.3 \pm 4.7$ years (range 0 to 19.7 years) after Fontan operation. In 2 patients atrial flutter occurred before the Fontan procedure and persisted after operation. In 4 patients atrial flutter was noted immediately after operation and recurred at a later time, whereas in others it developed as late as 19.7 years after operation.

Follow-up interval. The duration of follow-up was $8.7 \pm 3.9$ years (median 8.6 years) in patients with atrial flutter and $4.4 \pm 3.4$ years (median 3.4 years) in those without atrial flutter $(p<0.001)$. Atrial flutter continued to develop with time after Fontan operation (Fig. 1).

Age at operation. Patients in whom atrial flutter developed were older at the time of operation than those in whom it did not $(12.4 \pm 7.6$ vs $6.3 \pm 5.2$ years; $p<0.001)$. Atrial flutter developed earlier in those older than 10 years at Fontan operation $(p<$ 0.001, Fig. 2).

Postoperative hemodynamics. A postoperative cardiac catheterization was done in 157 (47\%) of the patients, 29 of 54 with atrial flutter and 128 of 280 without atrial flutter. The mean right atrial pressure 


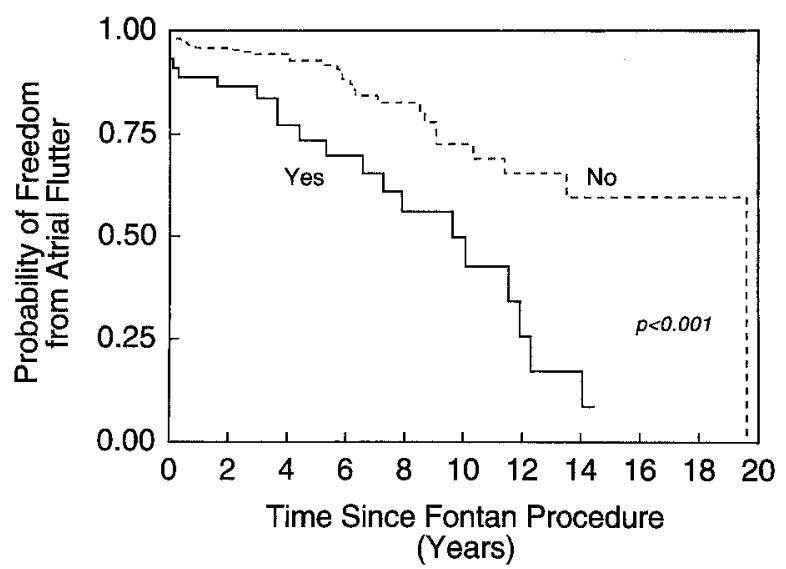

Fig. 3. Kaplan-Meier curves of freedom from atrial flutter comparing those with and without sinus node dysfunction.

was significantly greater in those with atrial flutter (15.1 \pm 3.9 vs $11.7 \pm 3.3 \mathrm{~mm} \mathrm{Hg} ; p<0.001)$.

Sinus node dysfunction. A total of 45 patients (13\%) had sinus node dysfunction noted during follow-up. Pacemaker implantation was done in $21(47 \%)$. The mean heart rate in those with sinus node dysfunction was $69 \pm 10$ beats/min as compared with $85 \pm 18$ beats $/ \mathrm{min}$ for those without sinus node dysfunction $(p<0.001)$. Predominant junctional escape rhythm was noted in $16(36 \%)$ with sinus node dysfunction. Among those with sinus node dysfunction, 20 (44\%) had atrial flutter, whereas only $12 \%$ of those without sinus node dysfunction had atrial flutter $(p<0.001)$. In those with sinus node dysfunction, atrial flutter developed earlier after the Fontan operation $(p<$ 0.001, Fig. 3).

Type of repair. Although there was a lower prevalence of atrial flutter in patients with a total cavopulmonary anastomosis, these patients were younger at the time of Fontan operation and had a shorter duration of follow-up. The development of atrial flutter in patients with an extracardiac conduit was similar to that in patients with a direct atriopulmonary anastomosis (Fig. 4).

Anatomic diagnoses. Time to atrial flutter was similar among the six anatomic diagnoses. However, atrial flutter was likely to develop sooner in those patients with left-sided atrioventricular valve atresia who received an atrial baffle directing left-sided pulmonary venous return to a right-sided atrioventricular valve $(p=0.007)$. In patients who had a

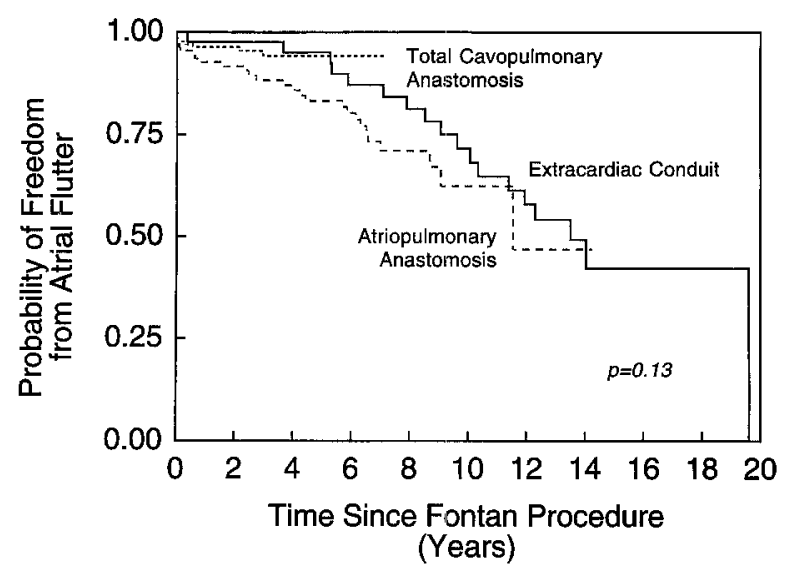

Fig. 4. Kaplan-Meier curves of freedom from atrial flutter as function of type of Fontan operation.

prior atrial septectomy or pulmonary artery reconstruction atrial flutter developed sooner $(p=0.004$ and $p<0.001$, respectively). Factors that were not found to be associated with an earlier incidence of atrial flutter included other previous palliative procedures and perioperative hemodynamics.

Survival/functional class. The presence of atrial flutter did not predict an increased incidence of death, transplantation, or takedown. Atrial flutter was less likely to occur in patients in New York Heart Association (NYHA) class I $(10 \%)$ than in those in class II or III ( $21 \%$ and $26 \%$, respectively, $p=0.02$ ). There were no patients in NYHA class IV in our study group.

Multivariate analysis. Analysis with a multivariate proportional hazards model suggested that an age at Fontan operation of 10 years or older (relative risk [RR] 3.0, 95\% confidence interval [CI] 1.7 to $5.2, p<0.001$ ), having had prior pulmonary artery reconstruction (RR $4.9,95 \%$ CI 2.0 to 11.9 , $p=0.001$ ), or requiring an atrial baffle to direct left-sided pulmonary venous drainage to a rightsided atrioventricular valve (RR 5.0, 95\% CI 1.9 to $13.0, p=0.001$ ) all predicted a shorter time to atrial flutter.

By a multivariate logistic regression model, atrial flutter was more likely to develop in patients if they were 10 years old or older at the time of Fontan operation $(p<0.001)$, had a prior atrial septectomy $(p=0.004)$, underwent the Fontan procedure in an earlier calender year (before 1988, $p=0.02$ ), or had a longer follow-up interval $(p=0.02)$. 


\section{Discussion}

The high prevalence of atrial flutter at follow-up was associated with an older age at operation, a longer duration of follow-up, and the presence of coexisting sinus node dysfunction. The $16 \%$ prevalence of atrial flutter detected in our patient population is consistent with reported intermediate and long-term results of other centers. ${ }^{1-6}$ The prevalence of atrial flutter increases with time after Fontan operation, and it is likely there will be an increasing number of patients in whom atrial flutter will develop in this patient population. In one patient in our study population atrial flutter developed almost 20 years after Fontan operation.

Weber and colleagues ${ }^{2}$ previously reported the continuous decline in the proportion of arrhythmiafree patients in a small series of patients after Fontan operation. The occurrence of late postoperative atrial arrhythmias that gradually increases with continued follow-up has also been described in patients who have undergone a Mustard or Senning operation for transposition of the great arteries. ${ }^{11-13}$

The presence of a region of structural or functional block within the atria is a substrate for atrial muscle reentry. ${ }^{14-20}$ The Fontan operation results in regions of structural block as a result of atrial incisions and multiple suture lines, as well as functional block from relative right atrial hypertension with right atrial dilation ${ }^{21}$ and hypertrophy. During electrophysiologic study after Fontan repair, Kurer, Tanner, and Vetter ${ }^{21}$ reported a high prevalence of abnormal sinus node function, prolonged atrial refractoriness, delayed intraatrial conduction, and inducible atrial arrhythmias.

In our population, there was a substantial proportion of patients with coexisting sinus node dysfunction and atrial flutter. This has been previously well described as the "tachycardia-bradycardia syndrome" in patients who have undergone operation. ${ }^{11-13}$ The frequent occurrence of sinus node dysfunction after Fontan operation is not surprising given the previous experience with the Mustard and Senning population. These atrial baffling operations are similar to the Fontan operation in that they require incisions and suturing in the region of the sinus node and its arterial supply. It has been speculated that bradycardia predisposes the heart to ectopic beats and prolonged refractoriness, which may provide the physiologic environment for atrial reentry. ${ }^{22}$ This concept is supported by the observation of improved control of atrial reentry tachycardia with antibradycardia pacing in some patients. ${ }^{23}$
Previous reports on factors that may be associated with the development of tachyarrhythmias after Fontan operation identified older age at Fontan operation, increased right atrial size and pressure, elevated preoperative pulmonary artery pressure, and depressed ventricular function as possible factors. ${ }^{3,4}$ In our population, elevated right atrial or pulmonary artery pressure measured at preoperative cardiac catheterization or by intracardiac catheters on the first postoperative day did not predict the development of atrial flutter. However, those patients with atrial flutter had an increased right atrial pressure at postoperative cardiac catheterization. Right atrial size was not examined in a systematic fashion, and therefore we were unable to evaluate this factor in our population. Clinical heart failure, as defined by NYHA class II or greater, was associated with an increased incidence of atrial flutter.

Older age at Fontan operation predicted the development of atrial flutter; however, this relationship was not linear. Although development of atrial flutter was more likely in those older than 10 years old at operation, there were no differences detected between the younger age groups. In comparing outcome of patients who underwent a Fontan operation before age 2 years with that of older patients, Weber and colleagues ${ }^{24}$ found that late arrhythmias occurred with equal frequency.

The introduction of the total cavopulmonary anastomosis and lateral right atrial wall tunnel has led some to postulate that this technique may result in a decreased incidence of atrial arrhythmias when compared with an atriopulmonary connection. It has been proposed that a smaller atrial mass is exposed to a chronic increase in systemic venous pressure and might be beneficial in preventing late-onset arrhythmias. $^{3,6,25}$ Although Balaji and associates ${ }^{6}$ reported a decreased incidence of early arrhythmias in the total cavopulmonary anastomosis group, no difference was detected in the incidence of late arrhythmias. A retrospective review of early Fontan procedure survivors reported that patients who had a direct atriopulmonary connection were more likely to have the development of atrial arrhythmias. ${ }^{10}$ However, that study grouped together a variety of supraventricular arrhythmias with different mechanisms. We found that the development of atrial flutter was similar over time in those who received repair with an extracardiac conduit or a direct atriopulmonary anastomosis, though initially atrial flutter appeared sooner in those with a direct atrial 
pulmonary anastomosis. Those with a total cavopulmonary anastomosis appeared to have a decreased incidence of atrial flutter; however, the follow-up for this group of patients was shorter.

Although a particular diagnosis was not associated with an increased incidence of atrial flutter, in those patients in whom extensive atrial baffling was used atrial flutter was more likely to develop. This finding may be related to more extensive operation within the atria or involvement of critical regions within the atria. This may also be reflected in the finding that atrial flutter was more likely to develop in patients who had a previous atrial septectomy, although atrial septectomies are frequently done in patients with left-sided atrioventricular valve atresia, some of whom also had complex intraatrial baffling.

Study limitations. The "true" incidence of postoperative atrial flutter is difficult to quantify. This study may overrepresent the percentage of patients in whom atrial flutter developed, because patients with symptoms may be more likely to seek medical attention and thus may have more recent follow-up. However, it is also possible that some of the late deaths were caused by an undetected episode of atrial flutter, thus underestimating the overall prevalence of atrial flutter. Furthermore, the group with the most recent surgical modification, the total cavopulmonary anastomosis, had the shortest duration of follow-up.

Nevertheless, we have shown that atrial flutter continues to develop with time after the Fontan operation. The high frequency of this complication further demonstrates the palliative nature of the Fontan circulation. The recent trend of performing the Fontan operation at a younger age may contribute to a decreased incidence of atrial flutter. Earlier identification of sinus node dysfunction with early institution of atrial pacing may also serve to decrease the incidence of atrial flutter in this group of patients. Finally, although the total cavopulmonary anastomosis appears to be associated with a decreased incidence of atrial flutter, continued prospective follow-up is necessary.

\section{REFERENCES}

1. Girod DA, Fontan F, Deville C, Ottenkamp J, Choussat A. Long-term results after the Fontan operation for tricuspid atresia. Circulation 1987;75:605-10.

2. Weber HS, Hellenbrand WE, Kleinman CS, Perlmutter RA, Rosenfeld LE. Predictors of rhythm disturbances and subse- quent morbidity after the Fontan operation. Am J Cardiol 1989;64:762-7.

3. Gewellig M, Wyse RK, de Leval MR, Deanfield JE. Early and late arrhythmias after the Fontan operation: predisposing factors and clinical consequences. Br Heart J 1992;67: $72-9$.

4. Peters NS, Somerville J. Arrhythmias after the Fontan procedure. Br Heart J 1992;68:199-204.

5. Driscoll DJ, Offord KP, Feldt RH, Schaff HV, Puga FJ, Danielson GK. Five- to fifteen-year follow-up after Fontan operation. Circulation 1992;85:469-96.

6. Balaji S, Gewellig M, Bull C, de Leval MR, Deanfield JE. Arrhythmias after the Fontan procedure: comparison of total cavopulmonary connection and atriopulmonary connection. Circulation 1991;84(Suppl):III162-7.

7. Kurer CC, Tanner CS, Norwood WI, Vetter VL. Perioperative arrhythmias after Fontan repair. Circulation 1990; 82(Suppl):IV190-4.

8. Mair DD, Hagler DJ, Julsrud PR, Puga FJ, Schaff HV, Danielson GK. Early and late results of the modified Fontan procedure for double-inlet left ventricle: the Mayo Clinic experience. J Am Coll Cardiol 1991;18:1727-32.

9. Hordof AJ, Edie R, Malm JR, Hoffman BF, Rosen MR. Electrophysiologic properties and response to pharmacologic agents of fibers from diseased human atria. Circulation 1976;54:774-9.

10. Gelatt M, Hamilton RM, McCrindle BW, et al. Risk factors for atrial tachyarrhythmias after the Fontan operation. J Am Coll Cardiol 1994;24:1735-41.

11. Kramer H, Rammos S, Krogmann O, et al. Cardiac rhythm after Mustard repair and after atrial switch operation for complete transposition. Int J Cardiol 1991;32:5-12.

12. Duster MC, Bink-Boelkens MTE, Wampler D, Gillette PC, McNamara DG, Cooley DA. Long-term follow-up of dysrhythmias following the Mustard procedure. Am Heart J 1985;109:1323-6.

13. Hayes CJ, Gersony WM. Arrhythmias after the Mustard operation for transposition of the great arteries: a long-term study. J Am Coll Cardiol 1986;7:133-7.

14. Rosenblueth A, Garcia-Ramos J. Studies on flutter and fibrillation: II-the influence of artificial obstacles on experimental auricular flutter. Am Heart J 1947;33:677-84.

15. Frame LH, Page RL, Hoffman BF. Atrial reentry around an anatomic barrier with a partially refractory excitable gap: a canine model of atrial flutter. Circ Res 1986;58:495-511.

16. Boyden PA, Frame LH, Hoffman BF. Activation mapping of reentry around an anatomic barrier in the canine atrium: observations during entrainment and termination. Circulation 1989;79:406-16.

17. Cronin CS, Nitta T, Mitsuno M, et al. Characterization and surgical ablation of acute atrial flutter following the Mustard procedure: a canine model. Circulation 1993;88(pt 2):461-71.

18. Boyden PA, Hoffman BF. The effects on atrial electrophysiology and structure of surgically induced right atrial enlargement in dogs. Circ Res 1981;49:1319-31.

19. Boyden PA. Activation sequence during atrial flutter in dogs with surgically induced right atrial enlargement: I- observations during sustained rhythms. Circ Res 1988;62:596-608.

20. Schoels W, Kuebler W, Yang H, Gough WB, El-Sherif N. A unified functional/anatomic substrate for circus movement atrial flutter: activation and refractory patterns in the canine 
right atrial enlargement model. J Am Coll Cardiol 1993;21: 73-84.

21. Kurer CC, Tanner CS, Vetter VL. Electrophysiologic findings after Fontan repair of functional single ventricle. J Am Coll Cardiol 1991;17:174-81.

22. Garson A. Medicolegal problems in the management of cardiac arrhythmias in children. Pediatrics 1987;79:84-8.

23. Rhodes LA, Walsh EP, Gamble WJ, Triedman JK, Saul JP. Benefits and potential risks of atrial antitachycardia pacing after repair of congenital heart disease. PACE Pacing Clin Electrophysiol 1995; 18:1005-16.

24. Weber HS, Gleason MM, Myers JL, Waldhausen JA, Cyran SE, Baylen BG. The Fontan operation in infants less than 2 years of age. J Am Coll Cardiol 1992;19:828-3.

25. Pearl JM, Laks H, Stein DG, Drinkwater DC, George BL, Williams RG. Total cavopulmonary anastomosis versus conventional modified Fontan procedure. Ann Thorac Surg 1991:52:189-96.

\section{ON THE MOVE?}

Send us your new address at least six weeks ahead

Don't miss a single issue of the journal! To ensure prompt service when you change your address, please photocopy and complete the form below.

Please send your change of address notification at least six weeks before your move to ensure continued service. We regret we cannot guarantee replacement of issues missed due to late notification.

\section{JOURNAL TITLE:}

Fill in the title of the journal here.

\section{OLD ADDRESS:}

Affix the address label from a recent issue of the journal here.

\section{NEW ADDRESS:}

Clearly print your new address here.

Name

Address

City/State/ZIP
COPY AND MAIL THIS FORM TO:

Journal Subscription Services

Mosby-Year Book, Inc.

11830 Westline Industrial Dr. St. Louis, MO 63146-3318
OR FAX TO:

314-432-1158

N/Mosby
OR PHONE:

1-800-453-4351

Outside the U.S., call

314-453-4351 